\title{
Cervical Agenesis: Two Case Reports with Review of Literature
}

\author{
Nayana Pathak ${ }^{1}$, Praveen Mohan ${ }^{2}$
}

\section{Abstract}

Cervical agenesis is an extremely rare congenital Mullerian duct anomaly of female genital tract. It is a complex surgical problem and management is still controversial. Failure to manage these patients correctly may be associated with long-term psychological, sexual, reproductive health damage and suffering. We have managed two cases of cervical agenesis with cryptomenorrhea.

\section{INTRODUCTION}

True incidence of cervical agenesis is unknown but review of literature list less than 200 cases since 1942 (1).According to American Fertility Society Cervical agenesis is classified as type IB mullerian anomaly(2). These defects are congenital, but usually present symptoms at the time of puberty. Patients have normal secondary sexual development and presentations are like primary amenorrhea, cyclical lower abdominal pain, mass per abdomen and history of recurrent surgeries.

\section{CASE 1}

An eighteen yrs old, unmarried girl presented to Gynae outdoor Deptt. With history of severe abdominal pain for last twenty days, secondary amenorrhea for three yrs and cyclical pain abdomen for the last six months. She underwent exploratory laparotomy, uterovaginal anastomosis with vaginoplasty in some other hospital at the age of thirteen yrs for cryptomenorrhea and had regular menstruation for six months following surgery. Her secondary sexual

\footnotetext{
1 Associate Professor

2 Professor and Head
}

Dept. of Obstetrics \& Gynecology, MMIMSR, Mullana, Ambala, India.

Correspondence: Dr. Nayana Pathak

E-mail:3261nayana@gmail.com

Competing interests: None characters were well developed. On per abdominal examination, a six into six $\mathrm{cm}$ mass was felt arising from the pelvis, more on the left side with evidence of guarding and rigidity. She was investigated thoroughly and all routine investigations werenormal. On Intravenous pyelography, left sided hydroureter with hydronephrosis was detected. Ultrasonography showed a bicornuate uterus with hematometra and left sided hematosalpinx. When examined under anesthesia, a blind ending vagina of $3 \mathrm{~cm}$ length and cervical agenesis was detected. As patient belonged to a poor socioeconomic group and wanted a permanent cure from the problem, exploratory laparotomy followed by total abdominal hysterectomy with

left side salpingo-oophorectomy, excision of right ovarian cyst and left sided ureteric stenting was done. Intraoperatively, 50-100cc dark altered blood was seen in pouch of Douglas with endometriotic implants over uterus, adenexae, POD, bladder and rectum. Bicornuate uterus of 8-10 wks size with hematometra, left sided hematosalpinx, endometriotic cyst of left ovary, right sided hydrosalpingx and simple cyst of right ovary was seen. (Fig1)

\section{CASE 2}

A fifteen years old girl presented with history of primary amenorrhea, cyclical pain abdomen and gradually increasing mass per abdomen for the last six months. She had history of laparoscopy one year back for acute pain abdomen and extensive endometriosis was diagnosed. After properexaminationandinvestigations, she diagnosed as a case of cervical agenesis with vaginal atresia. Exploratory laparotomy followed by total abdominal hysterectomy with

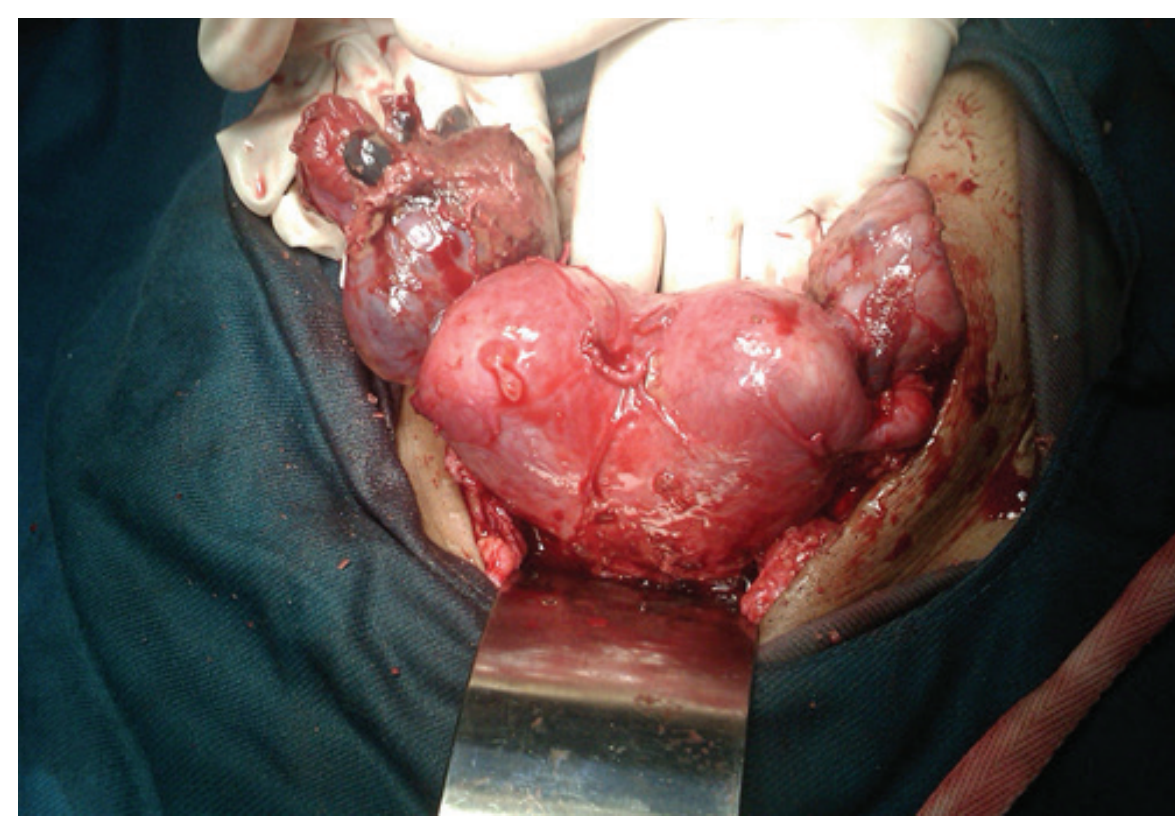

Figure 1: Uterus with left sided Tubo-ovarian mass \& right sided hydrosalpingx with cystic ovary 
bilateral salpingo-oophorectomy was done due to extensive endometriosis with complete cervical agenesis. Intraoperatively sixteen $\mathrm{wks}$ size enlarged uterus with bilateral endometrioma and hematosalpinx forming tubo-ovarian masses with extensive adhesion was seen. (Fig 2).

Postoperative period was uneventful in both patients and cervical agenesis was confirmed histopathlogically.

\section{DISCUSSION}

Congenital cervical atresia is a recognized clinical entity, but there is lack of uniformity in the literature regarding its classification and management. Embryologically, female reproductive tract develops from paired mullerian duct. Its complete formation and differentiation depends upon three phases of developmentorganogenesis, fusion (lateral as well as vertical) and septal resorption. Cervical atresia is considered as defect in the elongation of Mullerian Duct. At about 20wks of gestation, cervix forms as a condensation of stromal cell at a specific site around the fused Mullerian Duct and its differentiation is a complex process involves both mesodermal and endodermal tissue(3).The management of cervical anomalies is still controversial. Past and present articles are descriptive and difficult to assess and compare due to difference in sample size and variation in surgical techniques. Grimbizis (4) etal published the success of end to end cervico-cervical anastomosis in 116 cases of transverse cervical defect whereas Rober(5) etal have outlined the importance of cervical anatomy. In the literature role of conservative surgeries and successful pregnancy outcome have been reported with the help of assisted reproductive techniques but obstetrical complications have not been fully reported. In $50 \%$ cases cervical agenesis is associated with vaginal atresia and it is difficult to make a satisfactory fistulous tract in these types of cases. Complications like endometritis, Pelvic inflammatory disease, persistent pelvic pain, bowel and bladder injury, reobstruction, recurrent and severe pelvic infection,

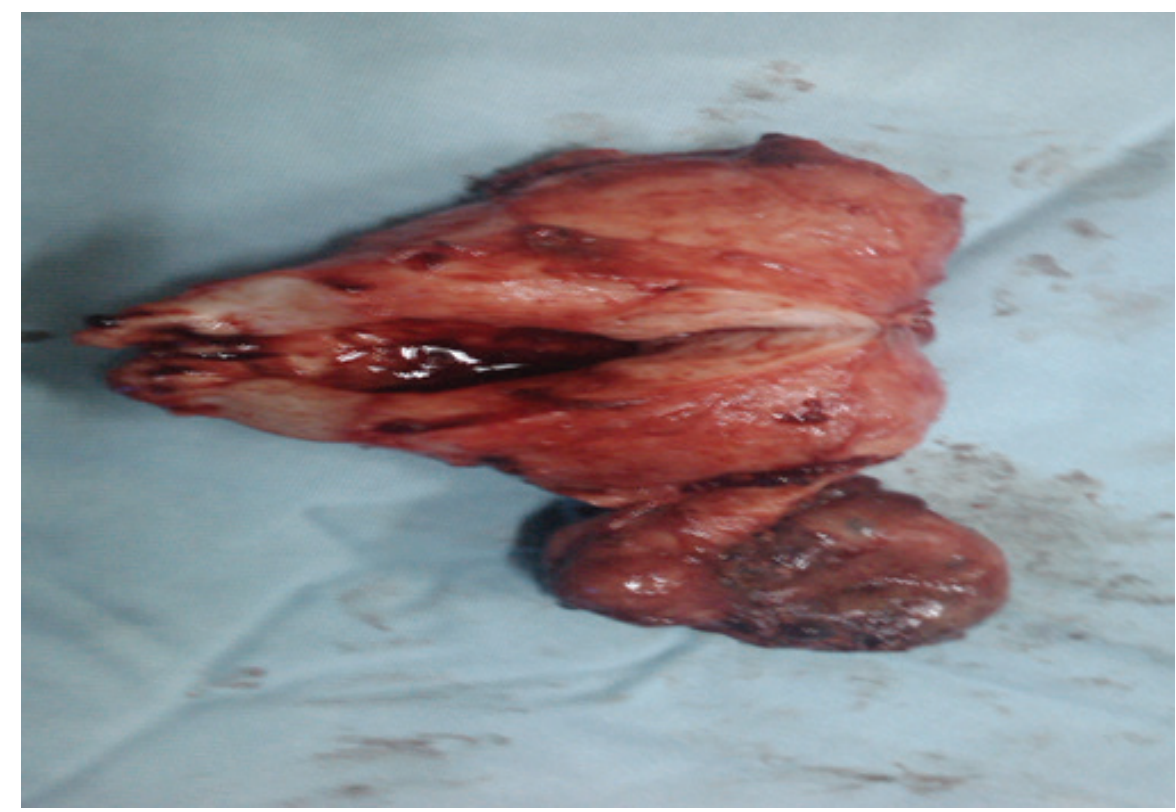

Figure 2: Cut section of uterus

repeated surgeries and even death has been reported in the literature following reconstructive surgeries. In Rock (1) etal series, success of reconstructive surgeries was documented in patients with cervical dysgenesis and all fourteen patients of cervical agenesis underwent hysterectomy. In all variants of cervical dysgenesis, successful outcome was obtained in only cervical obstruction group. In spite of documented success of reconstructive surgeries in the literature, it has challenges. Several factors may influence the surgical outcome: the size of created channel, the duration of stenting of the channel, the presence of rudimentary endocervical glands in the range of created channel, the presence of native vagina adjacent to created channel and the number of menses allowed to flow through the stented channel. Because the surgical approach for cervical cannulation is based only on several case reports, limited data are available regarding safety and efficacy to share with patients and relatives. A frank discussion with patient and her family regarding risk and morbidity of reconstructive surgeries is essential. In our opinion, reconstructive surgeries should be considered in selected patients and hysterectomy should always be offered as an option. Proper anatomical assessment, brief discussion regarding risk and morbidity associated with different surgical modalities and patient preference must be used for making treatment strategy, and then we can obtain a good surgical outcome.

\section{REFERENCES}

1. Rock JA, Roberts $\mathrm{CP}$, Jones HWJ. Congenital anomalies of the uterine cervix: lessons from 30 cases managed clinically by a common protocol. Fertil Steril 2010; 34: 1858-1863.

2. American Fertility Society (1988). The American Fertility Society classifications of Adnexal adhesions, distal tubal occlusion, tubal occlusion secondary to tubal ligation, tubal pregnancies, Mullerian anomalies and intrauterine adhesions. Fertil Steril 49,944-955.

3. UlfelderH, RobboyS. The embryologic development of the human vagina. Am J Obstet Gynecol 1976;126:766-769.

4. Grimbiziz GF, Tsalikis T, Mikos T, Et al. Successful end to end cervico-cervical anastomosis in a patient with congenital cervical fragmentation: case report. Hum Reprod 2004;5:1204-1210.

5. Roberts CP, Rock JA. Surgical methods in the treatment of congenital anomalies of the uterine cervix. Current Opinion in Obstetrics and Gynaecology 2011; 23:251-257. 